\title{
REPRESENTAÇÃO SOCIAL DA ENFERMAGEM DE REABILITAÇÃO: PENSAMENTO SOCIAL DOS ENFERMEIROS
}

\author{
Social representation of rehabilitation nursing: nurses' social thought \\ Representación social de la enfermería rehabilitadora: pensamiento social de las enfermeras
}

\author{
Isabel Oliveira*, Elisabete Ferreira**, Daniela Neves***, Ana Margarida Carvalho****, Mariana Sílvia*****, \\ Liliana Serrano******, Bruno Delgado*******
}

\begin{abstract}
RESUMO
Enquadramento: a qualidade em saúde é uma tarefa multiprofissional, na qual a enfermagem de reabilitação acrescenta valor pelo que, a compreensão a sua a representação social, poderá proporcionar contributos relevantes para aumentar a visibilidade da profissão e promover mudanças nas práticas e nos contextos de prática clínica. Objetivo: descrever a representação social da enfermagem de reabilitação para os enfermeiros. Metodologia: estudo qualitativo, exploratório e descritivo, numa amostra não probabilística acidental com 110 participantes, tendo como população alvo enfermeiros. A recolha de dados foi efetuada através de um teste de associação livre de palavras, por meio eletrónico, aos enfermeiros da rede de contatos de uma instituição de ensino superior. O tratamento de dados foi realizado através do software informático IRAMUTEQ. Resultados: $O$ núcleo central da representação social evidenciou as expressões autonomia, recuperação e cinesiterapia, reforçado pela primeira periferia independência, treino de marcha e cuidar. Domínios emergentes ou competências comuns, como o exercício físico e a gestão, foram menos relevantes para a estrutura da representação social. Conclusão: Não se conseguindo construir dentro da profissão um pensamento social que traduza o mandato social da enfermagem de reabilitação, a população poderá igualmente não o alcançar, o que poderá ter implicações na procura em cuidados.
\end{abstract}

Palavras-chave: enfermagem em reabilitação; psicologia social; enfermeiro

*RN, Prof. Adjunta da Escola Superior de Saúde Norte da Cruz Vermelha Portuguesa - https://orcid.org/0000-0001 6627-3907 - Contribuição no artigo: Study conception and design, Data analysis and interpretation, Drafting of the article, Critical revision of the article

RN, Enfermeira do Centro Hospitalar e Universitário de Coimbra - https://orcid.org/0000-0002-6286-4133 Contribuiçăo no artigo: Study conception and design, Data article, Critical revision of the article

${ }_{* * *}$ RN , Enfermeira do Centro Hospitalar e Universitário de Coimbra - https://orcid.org/0000-0002-3301-5757 Contribuição no artigo: Study conception and design, Data collection, Data analysis and interpretation, Drafting of the article, Critical revision of the article

****RN, Enfermeira do Centro Hospitalar e Universitário de Coimbra - https://orcid.org/0000-0003-0376-7250 Contribuição no artigo: Study conception and design, Data collection, Data analysis and interpretation, Drafting of the article, Critical revision of the article

de Coinbra de Coimbra - https://orcid.org/0000-0002-1101-3857 contribuiçaao no artigo: Study conception and design, Data article, Critical revision of the article

******RN, Enfermeira do Centro Hospitalar e Universitário de Coimbra - https://orcid.org/0000-0002-3621-0697. Contribuição no artigo: Study conception and design, Data collection, Data analysis and interpretation, Drafting of the article, Critical revision of the article ********PhD, Prof. Adjunto convidado na Escola Superior de Saúde Norte da Cruz Vermelha Portuguesa https://orcid.org/0000-0002-6847-1314 - Contribuição no artigo: Critical revision of the article

Como referenciar:

Oliveira, I., Ferreira, E., Neves, D., Carvalho, A.M., Silvia, M., Serrano, L., \& Delgado, B., (2021). Representação social da enfermagem de reabilitação: Pensamento Social dos enfermeiros. Revista de Investigação \& Inovação em Saúde, 4(1) 35-46. https:// doi.org/10.37914/riis.v4i1.124

\section{ABSTRACT}

Background: health quality is a multiprofessional task, in which rehabilitation nursing adds value, therefore understanding the social representation of rehabilitation nursing can provide relevant contributions to increase the visibility of the profession and promote changes in clinical practice practices and contexts. Objective: to describe the social representation of rehabilitation nursing for nurses. Methodology: qualitative, exploratory and descriptive study, in an accidental non-probabilistic sample with 110 participants, with nurses as the target population. The data collection was carried out through a test of free association of words, through electronic means, to the nurses in the network of contacts of a higher education institution. In data processing, we use the computer software IRAMUTEQ. Results: The central nucleus of the social representation evidenced the expressions autonomy, recovery and kinesitherapy, reinforced by the findings of the first periphery - independence, gait training and care. Emerging domains or common competencies, such as physical exercise and management, were less relevant to the structure of social representation. Conclusion: If it is not possible to build a social thought within the profession that translates the social mandate of rehabilitation nursing, the population may also not reach it, which could have implications for the demand for care.

Keywords: rehabilitation nursing; psychology, social; nurses

\section{RESUMEN}

Marco contextual: la calidad de la salud es una tarea multiprofesional, en la que la enfermería rehabilitadora aporta valor, por lo que entender su representación social puede aportar contribuciones relevantes para incrementar la visibilidad de la profesión promoviendo cambios. Objetivo: describir la representación social de la enfermería de rehabilitación para enfermeras. Metodología: Estudio cualitativo, exploratorio y descriptivo, en una accidental muestra probabilística con 110 participantes. La recolección de datos se realizó a través de una prueba de libre asociación de palabras, a través de medios electrónicos, a los enfermeros de la red de contactos de una institución de educación superior. En el tratamiento de los datos utilizamos el software informático IRAMUTEQ. Resultados: El núcleo central las expresiones autonomía, recuperación y kinesiterapia, reforzadas por la primera periferia: independencia, entrenamiento de la marcha y cuidado. Los dominios emergentes o las competencias comunes, como el ejercicio físico y la gestión, eran menos relevantes para la estructura de la representación social. Conclusión: Si no es posible construir un pensamiento social dentro de la profesión que traduzca el mandato social de la enfermería de rehabilitación, es posible que la población tampoco lo alcance, lo que podría tener implicaciones para la demanda de cuidados.

Palabras clave: enfermería de rehabilitación; psicología social; enfermera 


\section{INTRODUÇÃO}

Em saúde, a qualidade é uma tarefa multiprofissional, na qual os enfermeiros exercem um papel fundamental e, inerente à qualidade dos cuidados, assume-se existir hoje maior exigência técnica, científica e humana, sendo a especialização, cada vez mais, um imperativo que pretende dar resposta à crescente especificidade da necessidade em cuidados da população (Regulamento $\mathrm{n}$. 140/2019, de 6 de fevereiro). Neste sentido, a enfermagem de reabilitação acrescenta valor no processo de cuidados, maximizando o potencial de recuperação da pessoa, detendo o enfermeiro especialista em Enfermagem de Reabilitação (EEER) igualmente um conjunto de competências comuns, nomeadamente nos domínios da melhoria contínua da qualidade e do desenvolvimento das aprendizagens profissionais (Regulamento n.0392/2019, de 3 de maio; Regulamento n.o 140/2019, de 6 de fevereiro). A representação social, enquanto conjunto de suposições do conhecimento partilhado pelos indivíduos sobre um determinado objeto - no contexto deste estudo, a enfermagem de reabilitação - permite que esta seja descrita e explicada (De Rosa, Bocci, \& Dryjanska, 2018). A compreensão da representação social da enfermagem de reabilitação poderá proporcionar contributos relevantes para aumentar a visibilidade da profissão e promover mudanças nas práticas e nos contextos de prática clínica.

Neste contexto, foi objetivo deste estudo descrever a representação social da enfermagem de reabilitação para os enfermeiros.

\section{ENQUADRAMENTO/ FUNDAMENTAÇÃO TEÓRICA}

A representação social descreve a forma como os indivíduos adquirem e elaboram o conhecimento do mundo social através da partilha de suposições ou conceitos (De Rosa, Bocci, \& Dryjanska, 2018). Diz respeito a uma relação simbólica que valoriza a transformação do imaginário de uma comunidade e sintetiza o complexo entrelaçamento das relações culturais pelas quais as pessoas se relacionam entre si, num determinado contexto histórico e cultural (Rubira-Garcia, Puebla-Martínez, \& Gelado-Marcos, 2018). Sendo a representação social uma entidade dinâmica que desempenha um papel decisivo na configuração cognitiva e no comportamento dos indivíduos nos grupos, providencia um enquadramento holístico para a compreensão da apropriação e ação dos indivíduos (De Rosa, Bocci, \& Dryjanska, 2018; Rubira-Garcia, Puebla-Martínez, \& Gelado-Marcos, 2018). Apesar do conceito de representação social estar intimamente relacionado com a psicologia social (Rubira-Garcia, PueblaMartínez, \& Gelado-Marcos, 2018), a sua construção multidisciplinar tem feito crescer a sua utilização em outras áreas de conhecimento, nomeadamente na saúde e, concretamente, na enfermagem (Ferreira et al., 2019).

A enfermagem de reabilitação surge como uma das áreas de individualização das especialidades clínicas em enfermagem no contexto da evolução histórica da profissão, (Lei n.o 156/2015, de 16 de setembro). A individualização das especialidades clínicas em enfermagem surge como estratégia imprescindível, fundamentada na necessidade de uma atenção diferenciada e de qualidade, voltada para as 
necessidades da pessoa/família e prestada por enfermeiros especialistas devidamente certificados, no sentido de "...munir o enfermeiro de mais recursos para desenvolver respostas adaptadas em situações de grande complexidade, reconhecendo a centralidade da clínica na profissão de enfermagem" (Regulamento $\mathrm{n}$.으 168/2011, de 8 de março, p. 11120).

O enfermeiro especialista é assim detentor do um conjunto de competências específicas à sua área de intervenção, partilhando igualmente um conjunto de competências comuns: a responsabilidade profissional, ética e legal; a melhoria contínua da qualidade; a gestão dos cuidados e desenvolvimento das aprendizagens profissionais (Regulamento $\mathrm{n}$. 으 140/2019, de 6 de fevereiro). Estes domínios traduzem-se num nível elevado de capacidade de conceção, gestão e supervisão de cuidados e efetivo suporte ao exercício profissional especializado no contexto da formação, assessoria e investigação, envolvendo "as dimensões da educação dos clientes e dos pares, de orientação, aconselhamento, liderança, incluindo a responsabilidade de descodificar, disseminar e levar a cabo investigação relevante e pertinente, que permita avançar e melhorar de forma contínua a prática da enfermagem" (Regulamento $\mathrm{n}$. 140/2019, de 6 de fevereiro, p. 4744). Para além destas competências, o EEER é também detentor de um conjunto de competências específicas que decorrem das respostas humanas aos processos de vida e aos problemas de saúde, que se traduzem na capacidade para cuidar de pessoas com necessidades especiais, ao longo do ciclo de vida, em todos os contextos da prática de cuidados; de capacitar a pessoa com deficiência, limitação da atividade e/ou restrição da participação para a reinserção e exercício da cidadania e; o de maximiza a funcionalidade desenvolvendo as capacidades da pessoa (Regulamento n.ㅇ 392/2019, de 3 de maio). A especificidade da tomada de decisão permite que o EEER maximize o potencial funcional e autonomia do indivíduo, visando a independência e máxima satisfação da pessoa, utilizando para tal técnicas específicas de reabilitação, intervindo na educação dos clientes e pessoas significativas, no planeamento da alta, na continuidade dos cuidados e na reintegração das pessoas na família e na comunidade, assegurando, desta forma, o direito à dignidade e à qualidade de vida (Regulamento n.o 392/2019, de 3 de maio).

A abordagem à representação social da enfermagem de reabilitação justifica-se por diferentes razões. A representação social da enfermagem vai mudando ao longo do tempo e o estudo desta representação temse mostrado útil na compreensão das relações que se estabelecem entre o desenvolvimento da profissão e as políticas de saúde e o impacte da enfermagem na definição das políticas de saúde (Ravn et al., 2020). Além disso, a representação social é uma valiosa fonte de conhecimento que permite explorar como as pessoas e grupos participam na criação de sua identidade profissional percebida e, assim, refletindo e moldando a visão dominante da pessoa/família alvo dos seus cuidados e, em última análise, na construção da conceção do que é enfermagem (Ravn et al., 2020).

\section{METODOLOGIA}

Para responder ao objetivo deste estudo foi realizado um estudo de natureza qualitativa, com caráter exploratório e descritivo. A população em estudo foram os enfermeiros, recrutados para este estudo 
numa técnica de amostragem não probabilística por conveniência. Esta técnica de amostragem foi utilizada considerando a acessibilidade aos participantes, uma vez que foi utilizada a rede de contactos de uma instituição de ensino superior, que é formada por enfermeiros (antigos estudantes) que frequentaram e concluíram formação pré-graduada e/ou pósgraduada nesta instituição. Foram definidos como critérios de inclusão ser enfermeiro e consentir à participação neste estudo e como critério de exclusão ser EEER. Como instrumento de recolha de dados foi utilizado um questionário de autopreenchimento online com uma metodologia de teste de associação livre de palavras (TALP). O TALP, enquanto estratégia de recolha de dados, mostra-se particularmente relevante pois permite aceder, através de um estímulo indutor, à intrincada rede associativa de conteúdos evocados e representações do objeto indutor (Tavares, Brito, Córdula, Silva, \& Neves, 2014) e é frequentemente utilizado no contexto da investigação no domínio da Psicologia Social, com particular destaque nos estudos das representações sociais (Lopes et al., 2021). No TALP pede-se à pessoa que responda, com a primeira palavra que the venha à cabeça, a um estímulo indutor, que corresponde a uma série de palavras enunciadas pelo investigador. Ao serem solicitadas mais do que uma resposta para cada estímulo indutor, o participante é incentivado a hierarquizar as suas respostas (palavras ou expressões), de acordo com o grau de importância que Ihes atribui (Coutinho \& Bú, 2017). O questionário de recolha de dados foi enviado por correio eletrónico para o endereço eletrónico da rede de contactos da instituição de ensino superior e era composto por duas partes. A primeira com questões para a caracterização sociodemográfica dos participantes: sexo, tempo de exercício de funções como enfermeiro, existência de enfermeiro de reabilitação a exercer funções no seu serviço e a modalidade de afetação do enfermeiro de reabilitação ao serviço (se é elemento integrante da equipa ou se faz parte de uma equipa externa ao serviço). Na segunda parte do questionário surge o TALP, composto de 3 frases em que se solicitou ao participante que escrevesse as primeiras 5 palavras, por ordem de importância para o próprio, com seguintes termos indutores: "quando penso em enfermagem de reabilitação lembro-me de...", "quando penso no contributo da enfermagem de reabilitação no meu serviço, lembro-me de...", "quando penso nos cuidados de enfermagem de reabilitação, lembro-me de...".

A colheita de dados decorreu no período compreendido entre agosto a setembro de 2020, tendo sido recebido 110 questionários. Para a análise dos dados recolhidos utilizou-se o Interface de R pour les Analyses Multidimensionnelles de Textes et de Questionnaires (IRAMUTEQ), que consiste num programa que permite a análise dos dados recolhidos através do seu rigor estatístico (Camargo \& Justo, 2013). Este software possui a capacidade de identificar a quantidade de palavras inseridas tal como a frequência da palavra. Além disso, permite introduzir os dados até que estes saturem. Desta forma, revelase um instrumento de grande utilidade na análise dos dados qualitativos (Camargo \& Justo, 2013). Com esta análise, obtém-se uma imagem com quatro quadrantes que representam as quatro dimensões da estrutura da representação social (Abric, 2011). No primeiro quadrante, a zona do núcleo central, encontram-se as palavras com uma frequência maior 
que a média e evocadas nas posições mais elevadas, sendo que neste quadrante se encontram os elementos fundamentais que possuem ligações históricas, sociológicas e ideológicas e são consensualmente partilhados dentro do seio do grupo social e que, pela sua ligação à dimensão afetiva e ideológica, é onde se sustentam os estereótipos e crenças do grupo (Abric, 2011). Este quadrante evidencia a memória coletiva e o sistema de normas do grupo social, constituindo-se como a parte mais estável da representação social e mais resistente à mudança (Abric, 2011). O sistema periférico é a parte flexível da estrutura da representação e pode não ser partilhado consensualmente por todos os elementos do grupo. $O$ quadrante superior direito representa a primeira periferia e contém as palavras com frequência elevada, mas evocadas numa posição mais baixa, ou seja, evocadas mais tardiamente, constituindo-se como complemento primordial do núcleo central da representação. O quadrante inferior direito, a segunda periferia, indica respostas evocadas em último lugar e menor frequência e inclui respostas que são menos relevantes para a estrutura da representação social, traduzindo aspetos mais particularizados da mesma, e que são mais sujeitos a alterações de acordo com a representação dominante. Por último, a zona de contraste, que traduz as respostas com menor frequência, mas evocadas em primeiro lugar, sendo que estas respostas podem ser um complemento da primeira periferia e reforçar o núcleo central ou indicar a existência de um subgrupo que, ao valorizar consistentemente alguns elementos distintos da maioria, poderá ele próprio representar um núcleo central diferente (Abric, 2011).
Este estudo obteve parecer favorável da Comissão de Ética da instituição de ensino superior, com o número 25/2020.

\section{RESULTADOS}

No que se refere à caracterização dos 110 participantes verifica-se que a maioria ( $n=92 ; 85,3 \%)$ era do sexo feminino. Relativamente ao tempo de exercício de funções, este varia entre os 2 e 37 anos de serviço, sendo a média de 16,92 $\pm 9,58$ anos. Apurou-se igualmente que $59,1 \% \quad(n=65)$ dos participantes afirmou ter EEER no seu serviço e que em $64,5 \%$ ( $n=71)$ o EEER pertencia a equipa. Relativamente ao título profissional, 51,8\% ( $n=57)$ dos participantes eram enfermeiros de cuidados gerais e os restantes enfermeiros especialistas. Os dados recolhidos foram analisados através do programa IRAMUTEQ recorrendo-se à lematização dos mesmos. Dessa análise resultou a separação do corpus em 238 textos repartidos em 238 segmentos de texto. Nesses segmentos, foram identificadas 222 formas (redução as palavras com base nas suas raízes) e 825 ocorrências (resultado da análise de similitude que permite identificar o número de conexidade entre palavras). 0 número de hápax (palavras com frequência igual a 1) é 128 , o que equivale a $15,52 \%$ das ocorrências e $57,66 \%$ das formas. De forma a identificar a estrutura representacional a partir da frequência e ordem das evocações da matriz de dados utilizou-se uma análise prototípica.

A figura 1 mostra a distribuição das respostas pelo sistema central e periférico e pela zona de contraste. As respostas mais evidentes na zona do núcleo central das representações sociais dos enfermeiros sobre a 
enfermagem de reabilitação são autonomia, levante, equipa, incentivo e exercício físico. As recuperação, cinesiterapia, capacitar e qualidade de respostas treino, cooperação, função e intervenção vida. Quanto à zona da primeira periferia, destacam-se precoce são as mais evocadas na zona de contraste e as expressões independência, treino de marcha e em conjunto com as da primeira periferia reforçam a cuidar. Na segunda periferia surgem as palavras zona do núcleo central.

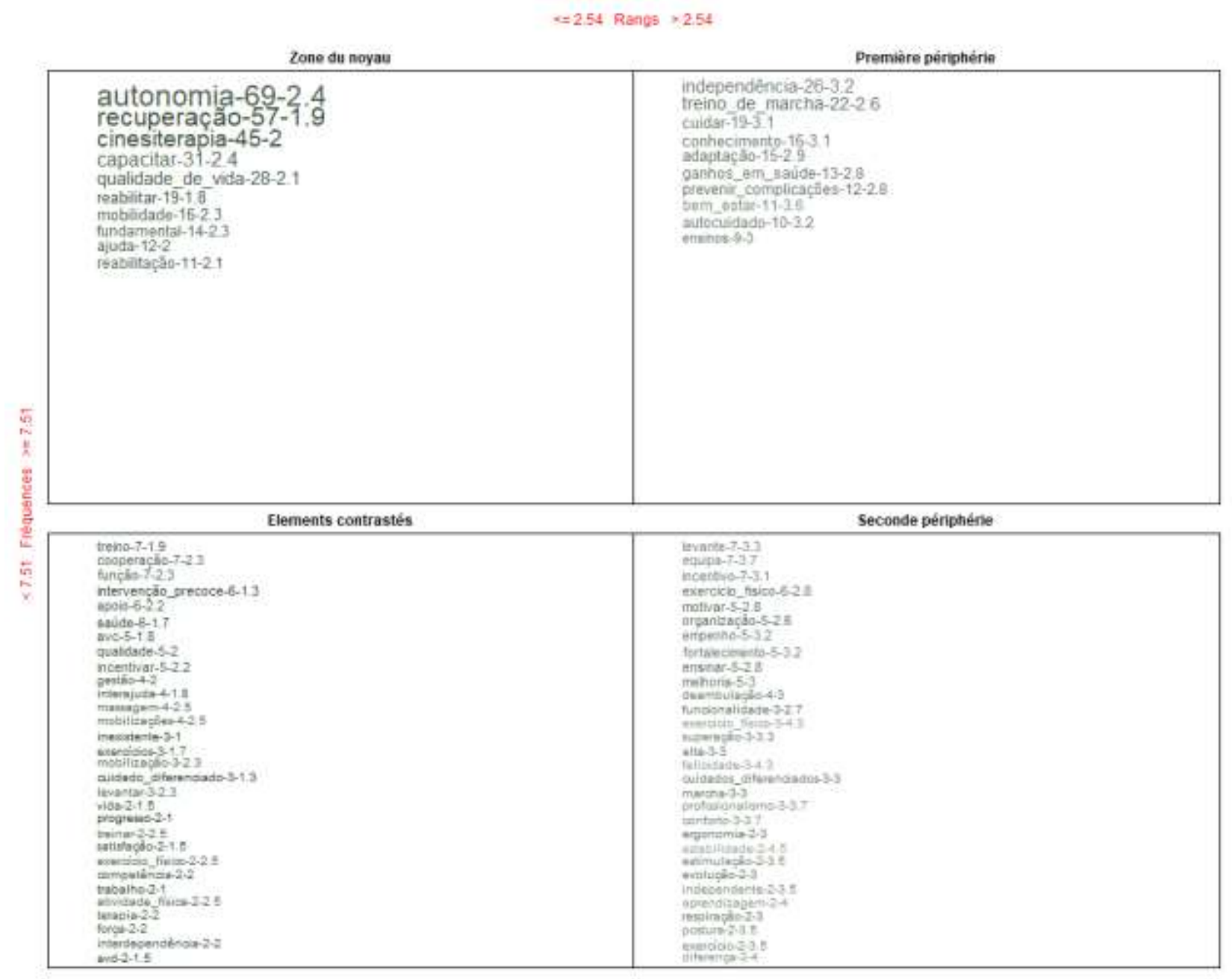

Figura 1

Análise Prototípica

Associado a esta análise, realizou-se uma análise de similitude ilustrada numa árvore que permitiu visualizar a relação entre as palavras e a sua conectividade dentro de cada classe e por outro lado a ligação entre as várias classes (figura 2). 


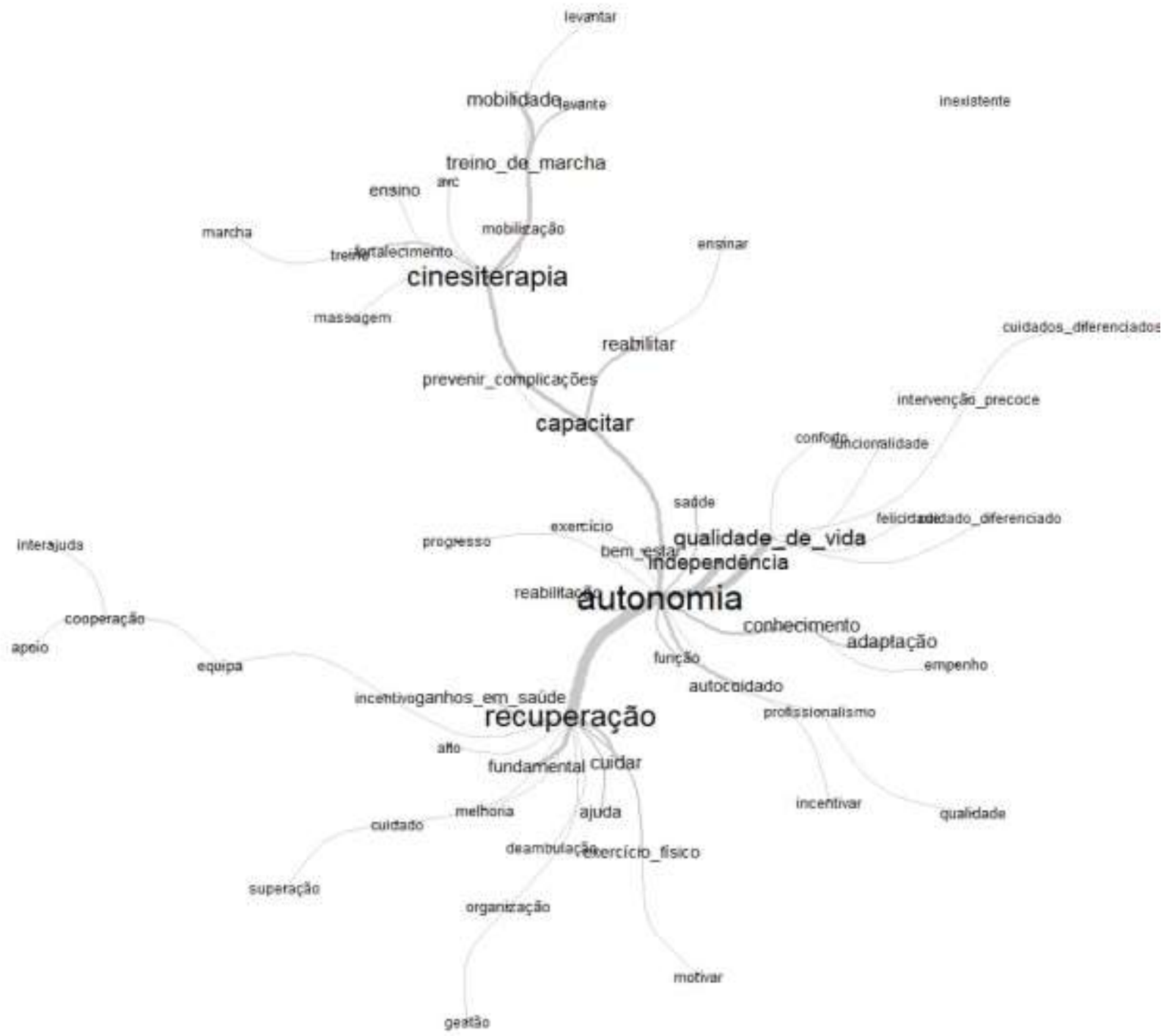

Figura 2

Árvore de Similitudes

Através da análise de similitude identificam-se que as expressões centrais, que estão ligadas por ramificações fortes entre si, são a autonomia, recuperação, cinesiterapia e capacitar. Verifica-se que a palavra autonomia está ligada qualidade de vida e independência. Já a palavra recuperação está ligada à palavra cuidar e ganhos em saúde. A palavra capacitar relaciona-se com a palavra cinesiterapia e reabilitar, principalmente. A palavra cinesiterapia está ainda ligada às palavras mobilidade e treino de marcha. Pelo método de nuvem de palavras, que as agrupa e organiza graficamente em função da sua frequência, das palavras mais evocadas pelos enfermeiros destacam-se a autonomia, recuperação, cinesiterapia, capacitar e qualidade de vida (figura 3). Nota-se na figura que as palavras são posicionadas aleatoriamente de tal forma que as palavras mais 
frequentes aparecem maiores que as outras, que a nuvem de palavras corrobora os resultados demonstrando assim, o seu destaque no corpus da expostos anteriormente.

análise da pesquisa. Deste modo, pode-se constatar

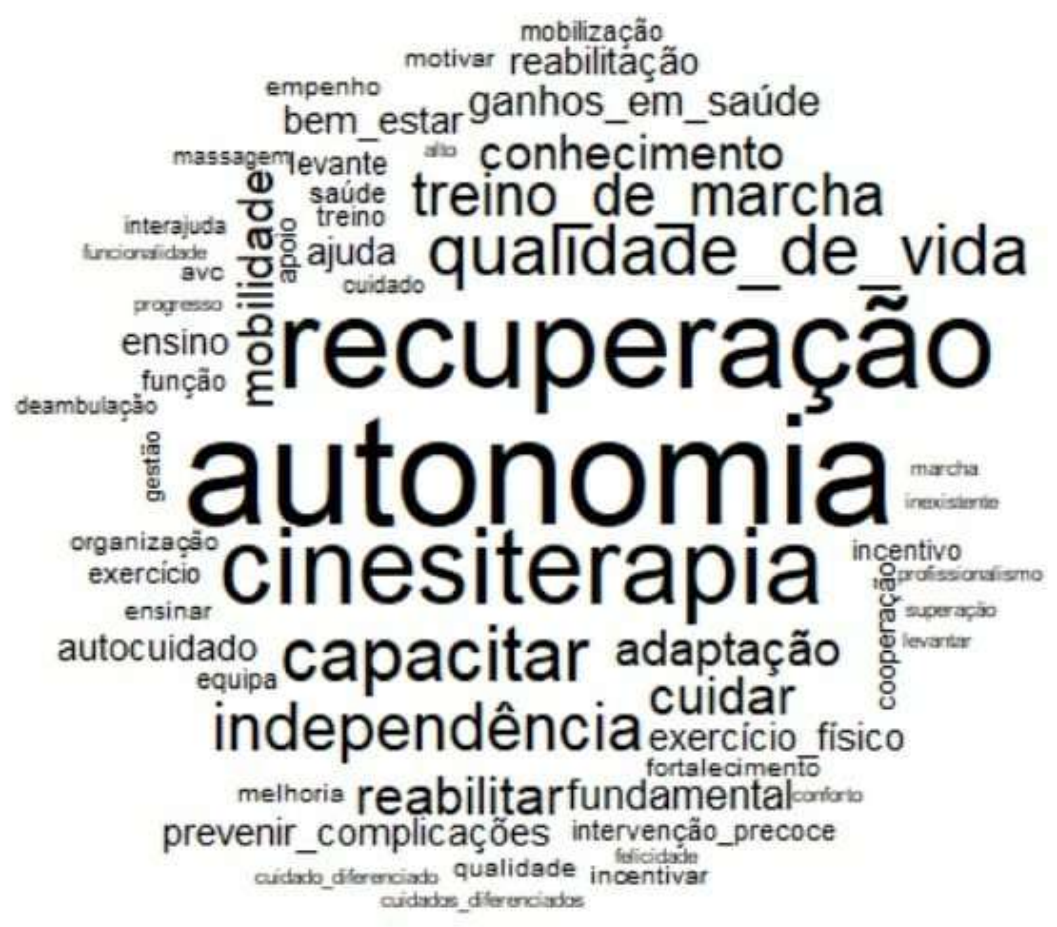

Figura 3

Nuvem de Palavras

\section{DISCUSSÃO}

Ao considerarem-se os resultados obtidos através da análise com recurso ao IRAMUTEQ, emergem essencialmente três expressões relativas à representação social da enfermagem de reabilitação para os enfermeiros: autonomia, recuperação e cinesiterapia, tendo como expressão mais evocada e com maior relevância no núcleo central, a autonomia. Tendo como referencial interpretativo destes resultados o perfil de competências do EEER, é percetível a ancoragem destes resultados ao conjunto de competências específicas do EEER, nomeadamente ao nível do cuidado à pessoa com necessidades especiais, ao longo do ciclo de vida, da capacitação da pessoa com deficiência, limitação da atividade e/ou restrição da participação para a reinserção e exercício da cidadania e na maximização da funcionalidade (Regulamento n.392/2019, de 3 de maio).

Outra das expressões encontrada no núcleo central é a expressão capacitar que releva para o papel do EEER 
enquanto facilitador da transição experienciada pela pessoa (Meleis, 2015), sugerindo o modelo concetual orientador da prática clínica do EEER. Para além disso, e atentando aos enunciados descritivos do Padrões de Qualidade dos Cuidados Especializados em Enfermagem de Reabilitação, as expressões identificadas no núcleo central sugerem uma centralidade da prática na reeducação funcional, que se distinguem dos Padrões de Qualidade dos Cuidados de Enfermagem (Ordem dos Enfermeiros, 2018), concorrendo para aquele que é o contributo singular que esta área específica do exercício profissional do enfermeiro traz para a pessoa. De facto, estes resultados vão ao encontro da definição do que é entendido como cuidados especializados de Enfermagem de Reabilitação, na sua especificidade e orientação para o processo de tomada de decisão do EEER no sentido da promoção da autonomia dos processos de readaptação (Ordem dos Enfermeiros, 2018). Estes resultados vão, em parte, ao encontro dos achados de Martins, Ribeiro e Silva (2018), que num estudo que pretendeu compreender a perceção do contributo dos EEER na concretização dos Padrões de Qualidade dos Cuidados de Enfermagem, concluí que este contributo está claramente orientado para a readaptação funcional, bem-estar e autocuidado. No entanto, uma dimensão que não fica evidente nos resultados e que traduz um foco de atenção relevante para a prática do EEER é a promoção da capacidade para o autocuidado, contrastando com os resultados deste estudo (Martins, Ribeiro, \& Silva, 2018; Ordem dos Enfermeiros, 2018) e que surge na primeira periferia numa posição de pouco relevo nas evocações dos participantes. Este achado sugere que nas representações sociais dos participantes da enfermagem de reabilitação não se identifica o um dos principais focos de atenção do EEER: o autocuidado (Ordem dos Enfermeiro, 2018). Também com pouco relevo para a estrutura das representações sociais surge na segunda periferia a expressão exercício físico. O exercício físico que tem ganho crescente importância na intervenção do EEER, evidenciando ganhos em saúde sensíveis aos cuidados de enfermagem de reabilitação (Gaspar, Ferreira, Vieira, Machado, \& Padilha, 2019), o que contrasta com as representações sociais dos participantes. Verifica-se que os achados da primeira periferia e zona de contraste reforçam os achados do núcleo central, que no seu conjunto denotam uma orientação das representações sociais para as questões relacionadas com a autonomia e a dimensão motora da intervenção do EEER (Regulamento n..392/2019, de 3 de maio), evidenciado pelas expressões cinesiterapia e treino de marcha, com frequência de evocação mais elevada. Não se identificam nos resultados expressões que traduzam a intervenção do EEER nos seus domínios de competências comuns (Regulamento n.․ 140/2019, de 6 de fevereiro). Apenas na zona de contraste existe a evocação da expressão gestão, com evocação elevada, mas baixa frequência. Os domínios da responsabilidade profissional, ética e legal, da melhoria contínua da qualidade e do desenvolvimento das aprendizagens profissionais não encontram eco nas evocações dos participantes. Estes resultados poderão ser compreendidos à luz dos resultados do estudo RN4cast Portugal, 2017-2018, tal como citados por Fronteira, Jesus e Dussault (2020), que traduzem ambientes de trabalho pouco favoráveis, especialmente nas vertentes da participação dos enfermeiros na Governação da Organização e na 
Gestão, Liderança e Apoio aos Enfermeiros, sugerindo que a perceção dos enfermeiros tem repercussões nas representações sociais da enfermagem de reabilitação, no domínio das suas competências comuns.

A teoria das representações sociais diz-nos que é, precisamente, nas interações sociais diárias e nos espaços formativos quotidianos que se constroem mutuamente, quer o objeto do cuidado, quer a identidade profissional e este conhecimento tem-se mostrado útil no domínio científico da enfermagem para facilitar o acesso à visão dos enfermeiros de diferentes dimensões da sua prática profissional (Ferreira et al., 2019). A construção da representação social poderá ser condicionada por fatores como: o paradigma de formação, local de trabalho e a própria cultura, o que significa que estes resultados poderão ser influenciados pelas características dos seus participantes (Moscovici, 1981). Importa salientar que a visão agora alcançada valoriza a experiência individual de cada um dos participantes num contexto coletivo, constituindo-se por isso num valioso contributo na amplificação do significado atribuído à enfermagem de reabilitação e que importará explorar mais aprofundadamente. A construção deste significado permite aos EEER a compreensão sobre a coerência entre as suas competências, as suas práticas e o mandato social da profissão.

\section{CONCLUSÃO}

Partindo do pressuposto que os termos mais frequentemente evocados e em ordem mais elevada assumem maior relevância no pensamento social dos participantes, ocupando o núcleo central, a representação social da enfermagem de reabilitação está vinculada à autonomia, recuperação e cinesiterapia, reforçada pelos achados da primeira periferia: independência, treino de marcha e cuidar. Domínios emergentes ou das competências comuns do EEER, como o exercício físico ou a gestão, foram menos relevantes para a estrutura da representação social. Estes achados sugerem que os participantes reconhecem através das práticas dos EEER apenas uma parte da total amplitude da sua intervenção. Para além disso, não fica evidente nos resultados aquilo que é a intervenção do EEER nos seus domínios das competências comuns, concretamente ao nível dos domínios da responsabilidade profissional, ética e legal, da melhoria contínua da qualidade e do desenvolvimento das aprendizagens profissionais.

Estes resultados contribuíram para ampliar a compreensão da visibilidade da enfermagem de reabilitação dentro da própria profissão, sendo que não se conseguindo construir dentro da profissão um pensamento social que traduza o mandato social da profissão, isto sugere que a população poderá igualmente não o alcançar. Daqui resultam implicações que poderão ter impacte, inclusivamente, na procura em cuidados por parte da população. Torna-se assim relevante compreender o que leva os participantes a não reconhecerem esta dimensão do exercício profissional especializado, sendo este um dos aspetos que merecerá atenção no desenvolvimento de investigação no futuro.

Como principal limitação deste estudo, a utilização da rede de contactos de uma instituição de ensino superior e a possibilidade de diferentes vivências dos participantes, que poderão ter influenciado os resultados. 
Salienta-se o carácter inovador desta pesquisa em Portugal e os contributos que traz para a compreensão da coerência entre o mandato social da profissão e os significados atribuídos à prática, dado que não foi identificada qualquer pesquisa desenvolvida sobre esta temática, impelindo a uma reflexão profunda sobre estas questões, não apenas na perspetiva da investigação, mas sobretudo na perspetiva da profissão.

\section{REFERÊNCIAS BIBLIOGRÁFICAS}

Abric, J. (2011). Les représentations sociales: aspects théorétiques. In J.Abric (Ed.), Pratiques Sociales et Représentations (pp. 15-46). Paris, France: Quadrigue/Presses Universitaires de France.

Camargo, B. V., \& Justo, A. M. (2018). Tutorial para uso do software IRaMuTeQ. Retirado de IRaMuTeQ: http://www.iramuteq.org/documentation/fichiers/tut oriel-portugais-22-11-2018

Coutinho, M.L., Bú, E. (2017). A Técnica de Associação Livre de Palavras sobre o Prisma do Software Tri-DeuxMots (version 5.2). Revista Campo do Saber, 3(1), 219$243 . \quad$ Recuperado de: https://periodicos.iesp.edu.br/index.php/campodosa ber/article/viewFile/72/58

De Rosa, A.M., Bocci, E., \& Dryjanska, L. (2018). The Generativity and Attractiveness of Social RepresentationsTheory from Multiple Paradigmatic Approaches in Various Thematic Domains:An Empirical Meta-theoretical Analysis on Big-data Sources from the Specialised Repository "SoReCom'A.S.de Rosa' @-library". Papers on Social Representations, 27(1), 6.1-6.35. Recuperado de: http://psr.iscteiul.pt/index.php/PSR/index

Ferreira, C., Lisboa, C., Moreira, D., Sousa, G., Teixeira, T., Príncipe, F., \& Mota, L. (2019). Transporte interhospitalar do doente crítico: representação social dos enfermeiros. Revista de Investigação \& Inovação em Saúde, 2(2), 29-38. https://doi.org/10.37914/riis.v2i2.55
Fronteira, I., Jesus, E.H., \& Dussault, G. (2020). A enfermagem em Portugal aos 40 anos do Serviço Nacional de Saúde. Ciência e Saúde Coletiva, 25(1), 272-282.https://doi.org/10.1590/141381232020251.28482019

Gaspar, L., Ferreira, D., Vieira, F., Machado, P., \& Padilha, M. (2019). O Treino de Exercício em Pessoas com Doença Respiratória Crónica Estabilizada: uma Scoping Review. Revista Portuguesa de Enfermagem de Reabilitação, 2(1), 59-65. https://doi.org/10.33194/rper.2019.v2.n1.08.4567

Lei $\mathrm{n} .0$ 156/2015, de 16 de Setembro. Diário da República n. 181 - I Série. Assembleia da República. Lisboa, Portugal.

Lopes, G., Rolim, I., Alves, R. S., Pessoa, T., Maia, E. R., Lopes, M., ... Queiroz, R. (2021). Social representations on diabetic foot: contributions to PHC in the Brazilian Northeast. Representações sociais sobre pé diabético: contribuições para Atenção Primária à saúde no Nordeste brasileiro. Ciência \& Saúde Coletiva, 26(5), 1793-1803. $\quad$ https://doi.org/10.1590/1413$\underline{81232021265.04702021}$

Martins, M. M., Ribeiro, O., \& Silva, J.V. (2018). O Contributo dos Enfermeiros Especialistas em Enfermagem de Reabilitação para a Qualidade dos Cuidados. Revista Portuguesa De Enfermagem De Reabilitação, 1(1), 22-29. https://doi.org/10.33194/rper.2018.v1.n1.04.4386

Meleis, A.I. (2015). Transitions Theory. Em M. Smith, \& M.E. Parker (Ed.), Nursing theories and nursing practice (4th Edition ed., pp. 361-380). F. A. Davis Company.

Moscovici, S. (1981). On social representations. Em J.P. Forgas (org.), Social Cognition - Perspectives on Everyday Understanding. London: AcademicPress.

Ordem dos Enfermeiros (2018). Padrões de Qualidade dos Cuidados Especializados em Enfermagem de Reabilitação. Lisboa, Portugal: Autor. Retirado de: https://www.ordemenfermeiros.pt/media/8192/pont o-4 regulamento-dos-padr\%C3\%B5es-qualidadeceer-1.pdf

Ravn, I. M., Beedholm, K., Frederiksen, K., Kvangarsnes, M., Foss, I. C., \& Knutsen, I. R. (2020). In search of the changeable: An analysis of visual 
representations of nursing in Norwegian and Danish professional nursing journals, 1965-2016. Nursing Inquiry, $27(3)$

https://doi.org/10.1111/nin.12340

Regulamento n.392/2019, de 3 de maio. Diário da República n. 085 - Il Série. Ordem dos Enfermeiros. Lisboa, Portugal.

Regulamento n.o 140/2019, de 6 de fevereiro. Diário da República n.o 26 - II Série. Ordem dos Enfermeiros. Lisboa, Portugal.

Regulamento n. $168 / 2011$, de 8 de março. Diário da República n. 47 - Il Série. Ordem dos Enfermeiros. Lisboa, Portugal.
Rubira-García, R., Puebla-Martínez, B., \& GeladoMarcos, R. (2018). Social Representations in Studying Information, Knowledge, and Mediations: A Critical Review. Social Sciences, 7(12), 256. http://dx.doi.org/10.3390/socsci7120256

Tavares, D.W., Brito, R.C., Córdula, A.C., Silva, J.T., \& Neves, D.A. (2014). Protocolo verbal e teste de associação livre de palavras. Ponto de Acesso, 8(3), 6479. Retirado de https://portalseer.ufba.br/index.php/revistaici 